\section{Cell-free joint regeneration}

\section{By Lauren Martz, Staff Writer}

Columbia University Medical Center researchers have identified a cellfree tissue-regeneration strategy that not only helps replace lost bone but also regenerates cartilage, a feat that has stymied previous joint replacement efforts. ${ }^{1}$

Treatments for the structural breakdown of bone and cartilage that occurs in osteoarthritis include total joint replacement with a metallic or synthetic prosthetic. The devices do a good job of mimicking joint function, but wear between the patient's bone and the device can lead to loosening of the prosthetic, infection and failure after $10-15$ years. ${ }^{2}$

Tissue regeneration using stem or progenitor cells represents an alternative to the devices but has its own risks, such as pathogen transmission, immune rejection and tumorigenesis. Other cell delivery challenges include preserving, maintaining and transporting the live cells, all of which drive up cost. ${ }^{3}$

Now, Columbia's Jeremy Mao and colleagues report a way to separate the benefits of tissue regeneration from the risks by using implantable bioscaffolds that contain growth factors. The goal is to encourage the patient's own cells to do the work, as the growth factors prompt endogenous chondrocyte- and osteoblast-like cells to migrate to damaged joints and regenerate both the cartilage and bone.

Mao is a professor at Columbia and director of the Tissue Engineering and Regenerative Medicine Laboratory at the medical center.

The researchers used laser scanning and computer-aided reconstruction to develop synthetic bioscaffolds made from poly$\varepsilon$-caprolactone and hydroxyapatite that structurally matched rabbit proximal humeral joints.

In skeletally mature rabbits, the team replaced surgically excised joint surfaces with either bioscaffolds infused with transforming growth factor- $\beta 3$ (TGFB3)-adsorbed collagen hydrogel, TGFB3-free bioscaffolds or no scaffolds.

Rabbits receiving the TGFB3-infused scaffolds recovered all weightbearing and locomotor functions within three to four weeks. In contrast, animals treated with TGFB3-free scaffolds had inconsistent recovery, and untreated animals limped throughout the study.

After the study, the team removed the bioscaffolds and found that the TGFB3-infused scaffolds had recruited $130 \%$ more chondrocytes than the
TGFB3-free bioscaffolds. The TGFB3-infused scaffolds also were covered in hyaline cartilage and integrated with the animals' own bone, and they regenerated subchondral bone and developed blood vessels.

In contrast, the TGFB3-free scaffolds had sporadic cartilage formation, and no cartilage formed in the joints of untreated animals.

The team, which also included researchers from the University of Missouri-Columbia and Clemson University, published its results in The Lancet.

"The advantage of this approach is the regeneration of articular cartilage covering the entire surface of the synovial joint without having to transplant cells, including stem or progenitor cells," Mao told SciBX. "Cartilage is one of the most recalcitrant tissues for regeneration."

"Academics and researchers from industry have been trying for years to develop better approaches to regenerate cartilage and synovial joint defects-it's a huge unmet need," said Jeffrey Karp, assistant professor of medicine at Harvard Medical School and co-director of regenerative therapeutics in the Department of Medicine at the Brigham and Women's Hospital.

Leo Snel, SVP of R\&D and protein chemistry at BioMimetic Therapeutics Inc., likes that the approach "leverages the effects of biological signals to mobilize endogenous cells into a repair site and therefore bypasses the need to harvest, expand, process and implant reparative stem or progenitor cells. The strategy significantly simplified the logistics involved in developing a clinically relevant approach, as the harvest of stem cells from the patient and the time and work required for their expansion, conditioning and preparation for implantation are not necessary." recombinant human platelet derived growth factor $\mathrm{BB}$ (PDGFBB) with a resorbable synthetic bone matrix to stimulate bone regeneration by attracting endogenous cells. The product is approved as an alternative to autografts in ankle, hindfoot and midfoot fusion surgeries in Canada. A premarket approval (PMA) is under FDA review.

According to Bridget Deasy, assistant professor in the Department of Orthopedic Surgery at the University of Pittsburgh and assistant professor at the McGowan Institute for Regenerative Medicine, "ex vivo manipulation of the stem cells makes other strategies risky because the chemicals introduced into the culture could change the identity of the cells, making them lose some of their stem cell properties and rendering them less effective, and could also induce DNA damage that could cause cancer."

\section{Next steps}

Columbia is seeking partners to pursue large animal studies and clinical trials of its bioactive scaffold.

Harvard's Karp suggested that the next set of preclinical studies should examine exactly how the bioscaffold functions. "More work is required to elucidate the mechanism for cell recruitment and to determine if the 
cells are homing from the bloodstream or migrating locally from the periwound compartment," he said.

"If we know where the cells are migrating from, we may be able to enhance the therapeutic efficacy for this application and others," said Karp. "If they are coming from the bone marrow, for example, we may be able to develop relevant model systems to screen for agents that target the right cell type to mobilize and home to diseased or damaged tissues more efficiently."

Snel noted that the five- to six-month-old rabbits used in the study were skeletally mature but still relatively young. As a result, he said, the animals' intrinsic repair capacities may not be the best proxy for the adults that would receive the therapy in a clinical trial.

"As we age, our intrinsic capacity for tissue repair diminishes. This is, in part, due to the decreased number of reparative cells and/or their reparative capacity," he said.

Snel added that any strategy that mobilizes endogenous reparative cells to an injury site will only work if patients have a sufficiently large pool of such cells.

Deasy noted that "one of the downsides of using the host cells is that they could be compromised in the patients to begin with. The patients are often older or have cells that are damaged simply due to the fact that they have the disease."

This could be a problem, she said, because the rabbit model does not give a good indication of the length of time for recovery in humans. "This will depend on the type of tissue that is damaged and the size of the tissue. Without a good supply of healthy endogenous cells, recovery could be very slow," Deasy said.

Although the Columbia bioscaffolds circumvent the potential side effects associated with cell therapies, the approach's use of growth factors carries its own risks.
"To achieve the desired tissue regeneration, the addition of a potent growth factor is required. This is potentially costly and may involve other untoward side effects," noted Molly Stevens, CSO of RepRegen Ltd.

RepRegen's StronBone bone graft substitute received European Conformity (CE) mark approval in the EU for hard tissue repair and regeneration. StronBone is a bioactive glass material that incorporates metal ions including strontium.

According to Snel, a growth factor-based strategy needs to show it can "elicit appropriate responses and not result in undesired effects such as exuberant or uncontrolled tissue growth, toxicities or malignancies."

Columbia has filed for a patent covering the technology.

Martz, L. SciBX 3(32); doi:10.1038/scibx.2010.975

Published online Aug. 19, 2010

\section{REFERENCES}

1. Lee, C.H. et al. Lancet; published online July 28, 2010; doi:10.1016/S0140-6736(10)60668-X

Contact: Jeremy J. Mao, Columbia University Medical Center, New York, N.Y. e-mail: jmao@columbia.edu

2. Lawrence, R.C. et al. Arthritis Rheum. 41, 778-799 (1998)

3. Muschler, G.F. et al. Tissue Eng. Part B Rev. 16, 123-145 (2010)

COMPANIES AND INSTITUTIONS MENTIONED

BioMimetic Therapeutics Inc. (NASDAQ:BMTI), Franklin, Tenn. Brigham and Women's Hospital, Boston, Mass.

Clemson University, Clemson, S.C.

Columbia University Medical Center, New York. N.Y.

Harvard Medical School, Boston, Mass.

McGowan Institute for Regenerative Medicine, Pittsburgh, Pa. RepRegen Ltd., London, U.K.

University of Missouri-Columbia, Columbia, Mo. University of Pittsburgh, Pittsburgh, $\mathrm{Pa}$. 